Disponível em

http://www.anpad.org.br/rac

RAC, Rio de Janeiro, v. 19, n. 2, art. 4, pp. 232-248, Mar./Abr. 2015

http://dx.doi.org/10.1590/1982-7849rac20151873

$(c))$ BY-NC

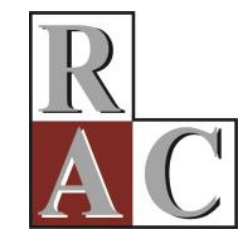

\title{
Responsabilidade Social Corporativa e Desempenho Financeiro das Empresas Brasileiras na Crise de 2008
}

\author{
Corporate Social Responsibility and Brazilian Firms' Financial Performance
}

Lilian Marques Freguete Fucape Business School

Valcemiro Nossa Fucape Business School

Bruno Funchal Fucape Business School

Artigo recebido em 07.03.2014. Última versão recebida em 19.08.2014. Aprovado em 24.08.2014. 


\title{
Resumo
}

A literatura aponta para alguns benefícios de longo prazo trazidos pela Responsabilidade Social Corporativa. Porém nada foi mencionado sobre seus potenciais benefícios de curto prazo, como, por exemplo, a performance da empresa em períodos de crise econômica e financeira, fenômeno reconhecido como sendo de curto prazo. Este artigo busca analisar a relação entre a prática de Responsabilidade Social Corporativa e o Desempenho Financeiro de empresas brasileiras durante um período da crise financeira de 2008. Utilizando modelos de diferenças-emdiferenças com dados em painel e considerando as empresas brasileiras que compõem a carteira ISE-BOVESPA, como proxy para empresas com práticas de Responsabilidade Social Corporativa, verificou-se que, durante crises, firmas responsáveis socialmente não têm um diferencial de desempenho financeiro em relação às outras firmas no mercado, apesar de ter-se encontrado um efeito significativo que a crise traz para as empresas em geral. Tal resultado não se alinha à teoria que prediz melhor desempenho econômico de empresas socialmente responsáveis ao perceber insignificância estatística para tal fator.

Palavras-chave: responsabilidade social corporativa; desempenho financeiro; crise.

\begin{abstract}
Literature on Corporate Social Responsibility points out some long-term benefits. However, the literature doesn't say much on its potential short-term benefits, such as how socially responsible firms react in a Financial-Economic Crisis, a well-known short-term phenomenon. This paper aims to analyze the relationship between Corporate Social Responsibility and firms' financial performance under a crisis scenario. Using a difference-in-difference regression model with panel data and companies listed on the Brazilian stock exchange (ISE-BOVESPA) as a proxy for Brazilian firms that implement Corporate Social Responsibility, we find that during the 2008 crisis, socially responsible firms didn't have significant differences in financial performance compared to non-ISE firms. Such results are not aligned with theories that argue in favor of Corporate Social Responsibility in order to boost financial performance.
\end{abstract}

Key words: corporate social responsibility; financial performance; crises. 


\section{Introdução}

Esta pesquisa teve como principal objetivo analisar um potencial benefício de curto prazo trazido pela Responsabilidade Social Corporativa: o desempenho financeiro das empresas brasileiras socialmente responsáveis durante um período de crise econômica.

Em geral, a Responsabilidade Social Corporativa (RSC - daqui em diante) está diretamente vinculada à sustentabilidade de longo prazo das empresas. A literatura sobre RSC (Du, Bhattacharya, \& Sen, 2010; Servaes \& Tamayo, 2013; T. Wang \& Bansal, 2012) tem revelado que empresas que se engajam socialmente criam efeitos positivos de longo prazo, como o aumento de valor da empresa, a construção de uma boa imagem, fortalecimento da relação entre stakeholders, redução de alguns tipos de riscos, etc. Note-se que tais efeitos gerados pela RSC, destacados pela literatura, são em geral efeitos positivos de longo prazo. Mas o que se pode referir sobre os potenciais efeitos de curto prazo?

Com relação aos impactos de curto prazo da RSC, a literatura destaca em sua maioria os custos incorridos pela empresa, monetários e não monetários, para a implementação das medidas relativas à RSC (T. Wang \& Bansal, 2012). Porém pouco se tem a comentar a respeito de seus potenciais benefícios.

Orlitzky, Schmidt e Rynes (2003) mencionam benefícios de curto prazo, por meio do comportamento das empresas, em períodos de crise. Os autores argumentam que investimentos em RSC são recursos capazes de fortalecer as firmas para enfrentarem períodos de crise, pois permitem melhor desenvolvimento de processos, mapeamento de habilidades, sistemas de informação, o que, em tese, aumenta seu preparo para enfrentar crises.

Assim, o foco do presente estudo foi investigar se a RSC também está associada a algum tipo de benefício de curto prazo. Aqui, considerou-se um benefício de curto prazo o comportamento da empresa em períodos de crise econômico-financeira, principalmente pelo fato de que crises são fenômenos associados exclusivamente ao curto prazo, ligados aos ciclos econômicos da economia (Haggard \& Willianson, 1994).

Assim, a principal contribuição do presente artigo à literatura de RSC é estender o debate de seus potenciais benefícios de acordo com o horizonte de tempo, e tal aspecto é diferente do que tem sido debatido, ou seja, focar nos benefícios de curto prazo. Sua contribuição secundária é em termos metodológicos. Neste estudo, foi utilizada uma abordagem quase-experimental, a qual leva em consideração problemas de causalidade para abordar o assunto. Além disso, os resultados aqui discutidos também contribuem de forma prática na decisão estratégica da empresa sobre sua responsabilidade social. Empresas mais sujeitas a oscilações em períodos de crise têm interesse em reduzir tais riscos por meio de certas políticas. Assim, estudar a contribuição da RSC para redução de riscos durante períodos de crise serve para dar suporte à decisão das empresas com esse tipo de foco.

Porém note-se que o debate de questões sociais tem sido, por décadas, um assunto amplamente discutido tanto nos meios acadêmicos quanto corporativos. Os diversos contratos explícitos e implícitos, assumidos pelas corporações com seus vários stakeholders (Cornell \& Shapiro, 1987; Freeman, 2010), fazem com que as empresas invistam cada vez mais em práticas socialmente responsáveis (Poddi \& Vergalli, 2009), haja vista o crescente número de novas certificações e índices de comprometimento com sustentabilidade que surgiram nas últimas décadas, tais como Dow Jones Sustainability Index, da Bolsa de Nova York, e o FTSE4Good, da Bolsa de Londres. Waller e Lanis (2009) assinalam o crescente interesse das empresas em declarar, voluntariamente, suas atividades de cunhos sociais e ambientais nos seus relatórios anuais a fim de mostrar uma boa imagem e reputação ante os diversos usuários.

Diversas são as motivações para a gestão orientada à Responsabilidade Social Corporativa (RSC). Para alguns administradores, direcionar recursos para fins do bem social pode funcionar como parte da estratégia elaborada a fim de as empresas alcançarem vantagens competitivas e sustentabilidade para seus empreendimentos. 
Os teóricos que defendem tal discurso argumentam que, com a prática de RSC, a empresa poderá atingir a otimização no uso dos recursos naturais e/ou matéria-prima de sua linha de produção, ter menor ônus com causas trabalhistas (bem como reduzir a rotatividade de funcionários), implementar mais inovações tecnológicas que aumentem a eficiência de seus processos, com isso, acarretando em menores custos de produção e, em consequência, um aumento de seu valor de mercado (Porter \& Linde, 1995; W. K. Wang, Lu, Kweh, \& Lai, 2014). No entanto, para outro perfil de administradores, pode ser apenas uma ferramenta de marketing nem sempre correspondente à real filosofia da empresa, comportamento este que Wagner, Lutz e Weitz (2009) denominam de Hipocrisia Corporativa, ou seja, o caso das empresas que afirmam praticar ações de responsabilidade social sem que estejam realmente fazendo. Isso leva a um distanciamento entre o discurso e a realidade praticada.

Outra linha de pensamento teórico defende que a preocupação com RSC significa um desvio do objetivo principal do administrador, qual seja o de maximizar o lucro para o shareholder. Para Friedman (1970), o único objetivo do administrador deve ser otimizar a lucratividade da firma. O exercício de RSC sinaliza conflito de agência já que não tem por finalidade o benefício do acionista.

Nesse sentido, tem-se a seguinte questão de pesquisa: Existe relação entre o exercício de Responsabilidade Social Corporativa e o desempenho econômico das firmas em cenários de crise econômica? A tentativa de investigar essa relação permite entender se existe um determinado tipo de benefício de curto prazo trazido pela RSC.

Para mensurar o potencial efeito de curto prazo da prática de RSC, foi utilizado o método quaseexperimental chamado de diferenças-em-diferenças, ou simplesmente diff-in-diff. A vantagem desse método é a possibilidade de explorar a adesão à prática de RSC como um evento que impõe, exogenamente, a percepção do público a uma melhora na responsabilidade social das empresas brasileiras e poder comparar com um grupo de empresas brasileiras que não seguem as mesmas práticas (ver Banerjee, Gertler, \& Ghatak, 2002; Coelho, De Mello, \& Funchal, 2012; Galiani, Gertler, \& Schargrodsky, 2005; Meyer, 1995). Assim, esse método busca comparar dois grupos, denominados tratamento e controle. O grupo de tratamento é formado pelas empresas que foram tratadas, isto é, implementaram a prática de RSC. O grupo de controle é formado por todas as outras empresas que não sofreram o tratamento. O diff-in-diff compara a mudança nos resultados no grupo de tratamento antes e depois da introdução da prática de RSC, com a mudança nos resultados no grupo de controle. Dessa forma, consegue-se levar em consideração, conforme este modelo empírico, todos os fatores não observados que tendem a afetar ambos os grupos ao logo do tempo.

Um ponto crucial está na definição das empresas tratadas, isto é, definir quem pratica a RSC. Nesse caso, foram consideradas empresas que compõem o Índice de Sustentabilidade Empresarial da Bovespa como proxy para firmas socialmente responsáveis, grupo de tratamento, a fim de serem comparadas às demais empresas listadas na Bovespa, grupo de controle. A implementação do diff-indiff foi realizada utilizando um modelo em painel com duplo efeito fixo, tanto na dimensão empresa quanto na dimensão tempo, de forma a controlar pelas características específicas invariantes no tempo e para controlar pelos ciclos da economia.

Os resultados encontrados apontam que não é possível afirmar que firmas que seguem as práticas de RSC têm um melhor desempenho financeiro em períodos de crise econômica, como sugerido por Orlitzky et al. (2003). Portanto, não se pode esperar que a prática de RSC apresente, como um potencial benefício de curto prazo, uma maior robustez financeira em períodos de crise.

Nas seções seguintes, apresentam-se a revisão da literatura sobre responsabilidade social corporativa, indicador de sustentabilidade brasileiro (ISE-BOVESPA) e crise econômico-financeira de 2008. Em seguida são descritas a metodologia de pesquisa, a análise discussão dos resultados e, por fim, as conclusões deste estudo. 


\section{Responsabilidade Social Corporativa}

Os estudos sobre Responsabilidade Social Corporativa têm sido, ao longo das últimas décadas, um tema constante nos meios acadêmicos. Isso, porém, ainda não conferiu um consenso quanto ao conceito e escopo do assunto. Para McWilliams e Siegel (2001), o termo se refere a ações para o bem social que vão além do cumprimento de leis, assumindo, assim, um caráter de voluntarismo.

Carroll (1979) relata que o conceito de responsabilidade social corporativa vem de longa data e já aparece na literatura acadêmica desde 1930. O autor sinaliza que o trabalho de Howard R. Bowen's, de 1953, intitulado Social Responsibilities of the Businessman, é considerado, por muitos, como o primeiro livro definitivamente dedicado ao assunto. Após tal publicação, muitos artigos surgiram e a década de 1960 foi um fértil período em publicações atinentes ao tema.

Diante dos diversos significados que o termo Responsabilidade Social Corporativa assume na literatura, Carroll (1979) classifica o assunto em quatro categorias de acordo com a motivação principal do ato: (a) Econômico: a principal função de empresas com fins lucrativos; (b) Legal: por estar inserida na sociedade, precisa enquadrar-se nos códigos e normas instituídos; (c) Ético: diz respeito a códigos relacionais implícitos que não estão, necessariamente, descritos na forma de lei; e, (d) Discricionário: que envolve ações de cunho estritamente voluntário.

O trabalho de Freeman (2010) é tido como seminal na fundamentação da teoria dos stakeholders (Mcwilliams \& Siegel, 2001). Para Freeman (2010), a abordagem convencional da teoria da firma contempla de forma sucinta as relações e contratos das corporações numa relação de oferta e demanda em que fornecedores, empregados e investidores proveem as necessidades da corporação, e esta, por sua vez, produz para os clientes os bens e/ou serviços explicitamente contratados.

$\mathrm{Na}$ teoria dos stakeholders, entretanto, os diversos grupos com interesses nas atividades da empresa (investidores, fornecedores, empregados, clientes, sociedade, governo e outros) exercem pressões sobre a corporação no sentido de satisfazer suas necessidades, tendo uma relação em que o fluxo dos direitos e deveres flui nos dois sentidos.

Os diversos stakeholders fazem pressões de toda natureza, e o administrador precisa lidar com estes fatores ao desenvolver seu planejamento estratégico. Donaldson e Preston (1995) argumentam que os estudos sobre a teoria dos stakeholders variam, muitas vezes, contraditoriamente, quanto às evidências e aos argumentos e apresentam pelo menos três formas distintas de tratar a RSC: (a) descritiva/empírica: em que os teóricos se baseiam na atestação de resultados obtidos por meio de experiências; (b) instrumental: em que os pesquisadores demonstram relação de causa e efeito nos comportamentos de RSC, recomendando-os (ou não) aos administradores a fim de alcançarem objetivos propostos; e (c) normativa: em que a teoria é usada para indicar a função da corporação moral e filosoficamente.

Com base na Teoria do Mercado Eficiente (Fama, 1970), Mackey, Mackey e Barney (2007), pressupõem que a informação de engajamento em atividades socialmente responsáveis refletirá no preço das ações e em seu valor de mercado. Os autores defendem que o investimento em ações de responsabilidade social pode ser entendido como um produto na medida em que os investidores procurem por tal característica na hora de escolher as ações que adquirirão. As práticas de responsabilidade social favorecem a legitimação dos stakeholders oferecendo uma imagem positiva da empresa e melhorando sua reputação. De acordo com a teoria dos stakeholders, a satisfação de vários grupos de stakholders é instrumento para o desempenho financeiro corporativo (Donaldson \& Preston 1995; Orlitzky, Schmidt, \& Rynes, 2003).

A adoção de estratégias de engajamento à RSC foi estudada por Tang, Hull e Rothenberg (2012). A pesquisa foi realizada por meio de uma análise longitudinal de dados de uma amostra de 130 empresas, de 1995 a 2007. Os resultados mostraram que empresas se beneficiam mais quando adotam estratégia de engajamento à RSC mais consistente, envolvendo dimensões. 
Entre os diversos estudos que buscam relacionar RSC e Desempenho Financeiro Corporativo (DFC), está o de López, Garcia e Rodriguez (2007), que tomam uma amostra de 110 firmas europeias, sendo 55 listadas no Dow Jones Global Index (o grupo de controle) e 55 listadas no Dow Jones Sustainability Index como grupo de tratamento, durante o período de 1998 a 2004. Percebe-se que as práticas de RSC têm impacto negativo no DFC, o que, para os autores, deve-se ao custo dos investimentos em adequação aos critérios de RSC, mas assinalam que os resultados positivos devem ser esperados em longo prazo.

Byus, Deis e Ouyang (2010) replicam o estudo de López et al. (2007), porém com empresas americanas, e ampliam o tamanho da amostra para 240 firmas. Ampliam, também, o período de 1999 a 2007. Os resultados obtidos se apresentam divergentes aos de López et al. (2007) e, estatisticamente, significantes para melhor desempenho das empresas com RSC quanto ao ROA e à margem bruta.

Ainda relacionando RSC e DFC, tem-se o trabalho de Mallin, Faraga e Ow-Yong (2014) no qual se detectou uma associação positiva e significante entre o Desempenho Financeiro e as dimensões da CSR, exceto para a dimensão ambiental. A pesquisa analisou uma amostra de 90 bancos islâmicos em 13 países.

Outro estudo que se utilizou do DJSI como proxy para assinalar práticas de RSC é o de Poddi e Vergalli (2009), que incluíram, em seu trabalho, mais outros dois índices de cunho semelhante, o Domini400 Social Index e o FTSE4 Good para sinalizar RSC. Como grupo de controle, usaram empresas listadas no Dow Jones Global Index. Assim como Byus et al. (2010), também, obtiveram resultados positivos ao relacionar RSC e DesemDFC. Porém observaram que o crescimento de práticas de RSC está fortemente relacionado ao desenvolvimento socioeconômico do país sede da firma e, portanto, tem sido mais notado na América do Norte e Europa do que nos países em desenvolvimento.

Orlitzky et al. (2003), diante da falta de consenso ou resposta elucidativa quanto à questão fundamental e reiteradas vezes estudada (qual a relação entre investimento em RSC e lucratividade), optaram por um estudo baseado na meta-análise. A meta-análise é uma técnica estatística que tem como objetivo integrar diversos resultados dos estudos que tratam de uma mesma questão de pesquisa, dessa maneira, fazendo uma revisão sistemática da literatura.

Assim, levantam os resultados de 52 estudos efetuados nas três décadas que o antecederam e os relacionam entre si. O objetivo da pesquisa é prover uma integração estatística das pesquisas anteriores que relacionam DFC a RSC com o intuito de avaliar a validade preditiva da teoria dos stakeholders na relação entre RSC e DFC. Os autores concluem que a RSC é, positivamente, correlacionada com DFC, pois a RSC aumenta competências gerenciais, contribui para o conhecimento organizacional sobre o mercado da empresa, quanto a questões sociais, ambientes políticos, tecnológicos, entre outros, aumentando, assim, a eficiência organizacional.

Pavie (2008) replica o estudo de Orlitzky et al. (2003), coletando estudos nos 10 anos subsequentes à pesquisa original (de 1998 a 2007) e verifica que os resultados permanecem idênticos, mesmo tendo sido a amostra ampliada para 112 estudos. Os resultados de Pavie (2008) também indicam uma relação positiva entre RSC e DFC, e tal relação ocorre de uma forma bidirecional, formando um círculo virtuoso, conforme também assinala Waddock e Graves (1997). Tal relação é fortemente moderada pela reputação da empresa.

Cochran e Wood (1984) criticam a utilização da análise de conteúdo dos relatórios contábeis e de índices de reputação quanto à prática RSC, cujos critérios subjetivos não se mostram muito claros.

Para Cochran e Wood (1984), o risco também é importante variável, pois, comparando o estudo de Moskowitz (1972) e de Vance (1975), percebeu que ambos utilizaram a mesma amostra de firmas, porém encontraram resultados opostos. A diferença estava no período da coleta em que os betas das firmas foram alterados. O primeiro estudo fora coletado em período de Bull Market, ou seja, de crescimento. Já no estudo de Vance (1975), a coleta foi realizada em período de Bear Market, ou seja, de queda. Esta diferença explicaria os resultados opostos das duas pesquisas. 
Ainda sobre os períodos analisados, Cochran e Wood (1984) argumentam que os estudos na área que o antecederam se utilizavam de períodos muito curtos, o que comprometia a ergodicidade e homocedasticidade das amostras, pois os resultados estariam sujeitos a pequenas variações. Os autores, em meados dos anos de 1980, já indicavam ser uma prática prudente administrar nos moldes da RSC, sob pena de terem, futuramente, altos custos de adequação às novas normatizações, com isso, evitando também futuras multas e punições quanto a danos trabalhistas, sociais e ambientais.

Com o desenvolvimento da literatura em termos metodológicos, Waddock e Graves (1997) encontram resultados positivos ao utilizar regressão múltipla para relacionar o desempenho financeiro e a prática de responsabilidade social corporativa. Para eles, existe um círculo virtuoso entre RSC e desempenho financeiro. Os recursos empregados nesta área podem representar vantagem competitiva, ao passo que tais práticas atraem melhores funcionários, assim como evitam custos com caras multas por problemas ambientais. A prática de RSC passa, então, a não mais ter um caráter apenas discricionário de práticas filantrópicas, mas se configura como estratégia para o alcance de vantagem competitiva.

\section{O ISE-BOVESPA}

O Índice de Sustentabilidade Empresarial, ISE-BOVESPA, é uma carteira de ações que tem por critério de inclusão a avaliação do desempenho ético, econômico, social e ambiental das empresas a ele submetidas (BM\&FBOVESPA, n.d.).

O ISE-BOVESPA foi criado em 2005, atendendo a uma demanda crescente no mercado mundial por investimentos responsáveis socialmente (SRI) e que tem como objetivo proporcionar ao acionista maior segurança quanto à rentabilidade a longo prazo porque as empresas nele listadas têm, por característica, menor vulnerabilidade a riscos econômicos, sociais e ambientais (Marcondes \& Bacarji, 2010). Também objetiva formar um benchmark quanto às boas práticas de gestão nos meios empresariais brasileiros (BM\&FBOVESPA, n.d.).

O ISE-BOVESPA é composto de até 40 empresas e é renovado a cada ano com base na adequação das empresas aos critérios de inclusão que contemplam características relativas à liquidez, à governança corporativa e às questões sociais e ambientais. Esses padrões são medidos por meio de questionários respondidos pelas empresas que possuem as 200 ações mais líquidas no exercício do ano anterior e que, após serem voluntariamente respondidos, são avaliados por um Conselho Deliberativo que seleciona a carteira seguindo as melhores classificações quanto:

1. Ao relacionamento com empregados e fornecedores

2. Ao relacionamento com a comunidade

3. À governança corporativa

4. Ao impacto ambiental de suas atividades

O ISE-BOVESPA, não obstante a sua recente criação, tem sido base para vários estudos nos meios acadêmicos brasileiros, muitos deles buscando, por meio de variadas ferramentas, identificar se há relação entre a RSC e o DFC.

Gomes e Gomes (2010) descrevem que os impactos provocados pela inclusão das empresas no ISE não se refletem só em relação ao desempenho financeiro, mas também quanto à reputação, ao disclosure ambiental, à regulamentação e qualidade e à formação de estratégia para alcance de vantagem competitiva. Os autores assinalam que as empresas do ISE, durante o período pesquisado (de 2005 a 2009), apresentaram resultados semelhantes aos apresentados pelo Ibovespa. 
Machado, Machado e Corrar (2009) buscaram averiguar se a rentabilidade média do ISEBOVESPA é, estatisticamente, semelhante à rentabilidade dos outros diversos índices da Bovespa e perceberam não haver diferença, estatisticamente significante, entre eles, no período de dezembro de 2005 a novembro 2007. Porém já assinalam que tal resultado pode ser em decorrência de que várias ações compõem, simultaneamente, a mais de um dos índices analisados.

Teixeira, Nossa e Funchal (2011) inovam na abordagem ao mudar o foco da rentabilidade, comum nos estudos anteriores, para a análise do risco e do endividamento, dessa maneira, comparando as empresas listadas no ISE a empresas não listadas. Os autores se utilizaram de regressão com dados em painel, levantados no período de 2003 a 2008, a fim de comparar o desempenho das ações antes e depois da criação do índice em 2005. E concluíram que a prática de RSC tem correlação negativa tanto com o risco quanto com o endividamento das empresas.

\section{A Crise Econômica Mundial de 2008}

A crise econômica mundial de 2008, também chamada Crise do Crédito ou Crise Americana, é entendida como um momento de ajuste dos sistemas econômico-financeiros que, segundo Minsk (1986), é endógeno aos mercados de molde capitalista democrático e sempre se segue aos períodos de grande pujança econômica.

Segundo Lal, Miller, Lieuw-Kie-Song, e Kostzer (2010), a crise de 2008 teve sua origem no mercado imobiliário americano, quando instituições financeiras, imprudentemente, ofertaram empréstimos com baixas taxas a mutuários sem credibilidade, lastro ou garantias (aqueles sem renda, postos de trabalho ou bens). Os títulos gerados destas operações foram comercializados no mercado financeiro subclassificado (subprime), porém ainda assim tomados por garantia nas negociações de créditos interbancários Mizen (2008).

O capitalismo ocidental, regido pelo que Gowan (2009) denomina de novo sistema de Wall Street, tem sua maior fonte de renda nos investimentos especulativos que, por conta da frouxa regulamentação dos sistemas financeiros, permitem uma alta volatilidade dos ativos financeiros. Isso consente que os investidores sejam capazes de inflacionar mercados emergentes, assim, objetivando os altos lucros, formando bolhas que se estouram quando da retirada maciça de ativos na corrida por liquidez.

Os investimentos são feitos, na maioria, com alto grau de alavancagem, pois, segundo Gowan (2009), apenas $10 \%$ do valor investido são patrimônios e os $90 \%$ restantes são dívidas. O modelo de precificação de ativos exercido por tais investidores é baseado em sofisticadas operações de securitização que transferem aos títulos uma característica nebulosa de valoração. $\mathrm{O}$ alto risco em operações que envolvem tais papéis é compensado pelos altos lucros que eles conferem. Tal engrenagem funciona bem até que, em um momento denominado Minsky, o mercado venha a corrigir este descolamento com traumáticos ajustes aos valores reais (Gowan, 2009).

Para Gowan (2009), a estrutura do mercado financeiro das décadas de 1990 e 2000 foi terreno fértil para a eclosão de uma crise de tamanha amplitude, tendo como importantes agentes: (a) o surgimento de um modelo de negociações de empréstimos; (b) a arbitragem especulativa dos preços dos ativos, com isso, causando a formação de bolhas; (c) a orientação para a maximização dos lucros; (d) o nascimento de um sistema bancário nebuloso, associado à criação de inovações financeiras; e (e) a centralização nos créditos derivativos que, associados a outras mudanças, mutuamente se reforçam culminando na sua desintegração em 2008.

As consequências da crise de 2008 foram extensivamente documentadas na literatura. A principal consequência foi a redução drástica dos empréstimos bancários ocorridos no período de crise (Ivashina \& Scharfstein, 2010). Empréstimos são vitais para o desenvolvimento da economia, pois é uma das principais fontes de recursos para viabilizar investimentos. Assim, outras consequências, como redução 
do crescimento econômico na maior parte dos países, mudanças em taxas de câmbio e aumento do desemprego também foram observadas (Coulibaly, Sapriza, \& Zlate, 2013; Rose \& Spiegel, 2012).

\section{Metodologia}

Este estudo objetivou identificar se existe relação entre a prática de Responsabilidade Social Corporativa e o Desempenho Financeiro Corporativo (DFC) de empresas listadas na Bovespa em um momento de crise no mercado financeiro mundial. Segundo a teoria da sinalização de Spence (1973), as entidades procuram emitir sinais a fim de inibir a assimetria de informação, desse modo, facilitando para o usuário a escolha entre as diversas ofertas. A inclusão em índices de sustentabilidade pode ser entendida como sinalização para a prática de Responsabilidade Social Corporativa.

Estudos anteriores, entre eles os de López et al. (2007), Lee e Faff (2009) e Byus et al. (2010), usaram, como critério para a identificação das empresas com comportamentos de RSC, a inclusão voluntária dessas empresas no Dow Jones Sustainability Index (DJSI).

No presente estudo, contextualizado no mercado brasileiro de ações, o termo sinalizador de RSC é a inclusão no ISE-BOVESPA, que se utiliza de critérios semelhantes aos do DJSI para a inclusão das ações em sua carteira. Note-se que a participação no ISE é o que permite diferenciar os grupos de tratamento, participantes do ISE, e controle, não participantes do ISE. Assim, torna-se possível aplicar a metodologia quase-experimental de diferenças-em-diferenças (Meyer, 1995) utilizando dados em painel e efeitos fixos. Essa metodologia, diferente de um modelo de regressão de Mínimos Quadrados Ordinários, permite a estimação direta da diferença entre diferentes grupos. Isso porque o modelo de diferenças-em-diferenças compara o comportamento de dois grupos de empresas - grupo de tratamento composto pelas empresas listadas no ISE e grupo de controle composto por empresas não listadas no ISE - em momentos distintos do tempo. Basicamente, o que se propõe é utilizar o comportamento do grupo de controle como um contrafactual do que seria o comportamento do grupo de tratamento, ou seja, representa o que teria acontecido às empresas listadas se estas não fossem listadas no ISE. Além disso, o efeito fixo controla por todas as características das empresas que são invariantes no tempo. Assim, sempre que se procurar comparar grupos em diferentes situações, o modelo de diferenças-emdiferenças é superior aos outros modelos de regressão, pois permite a estimação conjunta do efeito comparativo entre grupos e entre períodos.

Aqui, o que se visa estudar é o efeito do choque exógeno crise nos diferentes grupos. A vantagem dessa metodologia é a possibilidade de explorar como a adesão ao ISE influencia o comportamento durante um choque exógeno, determinado pela crise de 2008, comparativamente a um grupo de empresas que não segue as mesmas práticas, isto é, está fora do ISE.

Para implementar tal método, foi utilizado um modelo de regressão em painel cuja variável que captura a diferença de efeito da crise entre ambos os grupos é definida por uma interação de duas variáveis indicadoras: uma captura a primeira diferença, que é igual a 1 para a empresa listada no ISE e zero caso contrário; a outra captura a segunda diferença, sendo igual a 1 para o período de crise e zero caso contrário. O coeficiente estimado para essa variável que interage com ambas as variáveis indicadoras representa o efeito da crise sobre as empresas ISE relativamente às empresas Não-ISE.

A coleta dos dados foi feita na base de dados Economática, no período que compreende do ano 2005 (ano em que foi criado o ISE-BOVESPA) até 2010. O período crítico da crise financeira de 2008 é delineado a partir do terceiro trimestre de 2008 até o primeiro trimestre de 2009 , quando o preço das ações começou a voltar para o nível pré-crise.

Com uso da metodologia de López et al. (2007), tomou-se mais de uma variável dependente para medida de desempenho, sendo elas: 
1. O valor de mercado da empresa definido como o preço da ação multiplicado pelo número de ações da empresa. Tal variável foi escolhida a fim de perceber o impacto da crise no momento de seu ápice, pois o preço das ações é sensível a mudanças macroeconômicas como a crise de 2008.

2. O LAJIR (lucro antes dos juros e imposto de renda) como um indicador financeiro de lucratividade da empresa.

3. O market to book que indica a valorização das ações da empresa e é calculado dividindo-se o preço da ação no mercado pelo seu valor contabilizado.

Como variáveis explicativas, a exemplo de López et al. (2007), tomou-se como proxy para tamanho da empresa a variação dos Ativos Totais; como proxy para endividamento, a divisão da Dívida Bruta pelo Ativo. Ainda conforme López et al. (2007), levantou-se como variável explicativa a variação da receita.

Lang e Maffett (2011) evidenciaram que a gestão com alto padrão de transparência quanto aos relatórios contábeis, escolha dos auditores, gerenciamento dos resultados e precisão das previsões estão positivamente correlacionados à liquidez de mercado das ações e consequentemente ao valor da firma. À luz deste estudo, foi inserida a variável explicativa liquidez de mercado no modelo.

Por fim, foram criadas as variáveis indicadoras: (a) Dummy ISE, que indica as empresas tratadas, ou seja, as empresas que compõem o ISE recebem o valor 1; e as demais, o valor 0; (b) Dummy Crise, que indica o período de crise, sendo igual a 1 os períodos entre $4^{\circ}$ trimestre de 2008 e o $1^{\circ}$ trimestre de 2009; e (c) Dummy ISExCrise, que representa a variável de análise de diferenças-em-diferenças, e é definida pela interação entre as duas anteriores, demonstrando o desempenho das empresas com que estão no ISE em comparação com as demais durante o período de crise.

A amostra foi winsorada ${ }^{(1)}$ com o intuito de eliminar os outliers e, assim, obter uma amostra mais homogênea validando os pressupostos. Em todas as variáveis (exceto as dummys), foi utilizada a fração para ajuste das observações a um nível de $\mathrm{p}=0.025 \mathrm{em}$ cada cauda. O logaritmo natural foi utilizado em todas as variáveis, pois observou-se na amostra um desvio padrão elevado, mesmo após os dados serem winsorados.

Para implementar o modelo de diferenças-em-diferenças foi utilizada uma regressão em painel com duplo efeito fixo, melhor se adapta a estudos longitudinais com múltiplas variáveis cujas unidades se repetem ao longo dos diversos cortes transversais. O efeito fixo de cross-section é utilizado para capturar as características não observáveis invariantes no tempo, enquanto que o efeito fixo de tempo é utilizado para capturar elementos comuns a todas as empresas que são invariantes ao longo tempo.

Levantadas as variáveis descritas, foi proposta a seguinte equação de regressão:

$$
\begin{aligned}
& Y_{i t}=\alpha_{i}+\psi_{t}+\beta_{1} \text { crisexise }_{i t}+\beta_{2} \text { crise }_{i t}+\beta_{3} \text { ise }_{i t}+\beta_{4} \ln \text { wativo }_{i t} \\
& \beta_{5} \ln \text { wreceita }_{i t}+\beta_{6} \ln \text { wdiv_at }_{i t}+\beta_{7} \ln \text { wliquidez }_{i t}+\varepsilon_{i t}
\end{aligned}
$$

Onde:

$\mathbf{Y}_{\mathbf{i t}}=$ variáveis dependentes: Logaritmo natural do lucro antes dos juros e do imposto de renda (lnwlajir), o logaritmo natural do valor de mercado (lnwvm) e o logarítimo natural do market to book.

$\boldsymbol{\alpha}_{\mathrm{i}}=$ Efeito fixo de empresas.

$\psi_{t}=$ Efeito fixo de tempo.

$\boldsymbol{\beta}_{\mathbf{j}}=$ coeficientes, onde $j=1 \ldots 7$. 
$\mathbf{I s e}_{\mathbf{i t}}=$ variável dummy, em que recebe o valor 1 se a empresa (i) no trimestre (t) pertence ao ISE e 0 se não pertence.

Crise $_{\text {it }}=$ variável dummy, em que recebe o valor 1 se a empresa (i) no trimestre (t) está presente no período da crise $\left(4^{\circ}\right.$ trim. de 2008 e $1^{\circ}$ trim. de 2009$)$ e 0 os demais períodos coletados na amostra.

Isexcrise $_{\mathbf{i t}}=$ variável dummy que captura o efeito do diff-in-diff, isto é, a diferença dos grupos em períodos de crise. É representada pela interação entre as duas dummys anteriores, captando as empresas (i) no trimestre (t) que pertencem ao ISE e passam pela crise, recebendo o valor $1 \mathrm{e}$ recebendo 0 as demais empresas.

Lnwreceita $_{\text {it }}=$ variável logaritmo natural da receita líquida da empresa (i) no ano (t).

Lnwdiv_at $_{\mathbf{i t}}=$ variável logaritmo natural da dívida bruta do ativo da empresa (i) no ano (t).

Lnwativo $_{i t}=$ variável logaritmo natural do ativo da empresa (i) no ano (t).

Lnwliquide $_{i t}=$ variável logaritmo natural da liquidez de mercado da empresa (i) no ano (t).

$\boldsymbol{\varepsilon}_{\mathbf{i t}}=$ termo do erro estocástico da regressão.

Para corrigir os problemas de heteroscedasticidade e autocorrelação serial, comuns nesse tipo de dados, utilizou-se uma matriz de variância e covariância robusta a ambos os problemas, onde tal a matriz é consistente por construção.

\section{Análise dos Resultados}

A matriz de correlação pode ser utilizada como base para a escolha das variáveis que serão inseridas na equação do modelo. O ideal é que a variável explicativa principal seja altamente correlacionada com as dependentes, porém o que se observa é que a Dummy ISE tem fraca correlação com o LAJIR e valor de mercado, ainda que positiva.

Nas Tabelas 1 e 2, são mostradas as principais estatísticas descritivas das variáveis e sua matriz de correlação.

Tabela 1

Estatística Descritiva das Variáveis

\begin{tabular}{lccccc} 
& Observações & Média & Desvio Padão & Mínimo & Máximo \\
\hline Market-to-book (x) & 6311 & 2,09 & 2,64 & 0 & 13,6 \\
LAJIR (R\$ milhares) & 6901 & 102.776 & 211.944 & 41 & 1.006 .893 \\
Valor de Mercado (R \$ milhares) & 6426 & 4.010 .121 & 8.582 .254 & 4853 & 45.300 .000 \\
Ativo (R \$ milhares) & 7810 & 4.975 .626 & 1.090 .000 & 2567 & 5.910 .000 \\
Liquidez (milhares de negócios) & 9072 & 0,14 & 0,37 & 0 & 1,79 \\
Dívida/Ativo(x) & 7224 & 26,02 & 24,96 & 0 & 125,6 \\
Receita (R \$ milhares) & 6901 & 581.809 & 1.123 .178 & 0 & 5.348 .134 \\
ISE & 9072 & 0,067 & 0,25 & 0 & 1 \\
\hline
\end{tabular}

Nota. Fonte: Elaborada pelos autores. 
Tabela 2

Matriz de Correlação das Variáveis

\begin{tabular}{|c|c|c|c|c|c|c|c|c|c|c|}
\hline \multirow[b]{2}{*}{ lnwmvbv } & \multicolumn{3}{|c|}{ lnwmvbv lnwlajir lnwvm } & \multicolumn{3}{|c|}{ lnwativo lnwliquidez lnwdiv_at } & \multicolumn{2}{|c|}{ lnwreceita ise } & \multirow[t]{2}{*}{ crise } & \multirow[t]{2}{*}{ isexcrise } \\
\hline & 1,000 & & & & & & & & & \\
\hline lnwlajir & 0,2397 & 1,000 & & & & & & & & \\
\hline $\operatorname{lnwvm}$ & 0,4737 & 0,8231 & 1,000 & & & & & & & \\
\hline lnwativo & 0,1844 & 0,8408 & 0,8822 & 1,000 & & & & & & \\
\hline lnwliquidez & 0,2669 & 0,5325 & 0,6344 & 0,5549 & 1,000 & & & & & \\
\hline lnwdiv_at & 0,0385 & 0,0927 & 0,0148 & 0,0673 & 0,0203 & 1,000 & & & & \\
\hline lnwreceita & 0,2937 & 0,7841 & 0,8044 & 0,8370 & 0,4955 & 0,0858 & 1,000 & & & \\
\hline ise & 0,0904 & 0,3618 & 0,3860 & 0,3966 & 0,3591 & 0,0621 & 0,3433 & 1,000 & & \\
\hline crise & $-0,0795$ & 0,0061 & $-0,0476$ & 0,0119 & $-0,0089$ & 0,0064 & 0,0060 & $-0,0135$ & 1,000 & \\
\hline isexcrise & $-0,0016$ & 0,0897 & 0,0857 & 0,1080 & 0,0887 & 0,0210 & 0,0885 & 0,2700 & 0,3027 & 1,000 \\
\hline
\end{tabular}

Nota. Fonte: Elaborada pelos autores.

É possível notar que a variável independente ativo total (lnwativo) é altamente correlacionada com as variáveis dependentes LAJIR - lucro antes dos juros e imposto de renda (lnwlajir) $r=0,841$, com o valor de mercado (lnwvm) apresentando $\mathrm{r}=0,882$. Também a variável explicativa receita (lnwreceita) tem alto grau de correlação com as variáveis dependentes valor de mercado (lnwvm) com r=0,80 e com o LAJIR (lnwlajir) $r=0,78$. Tais resultados são desejáveis porque se espera que o modelo explique de forma satisfatória a relação entre elas.

O modelo de diferenças-em-diferenças foi estimado utilizando uma regressão em painel com duplo efeito fixo (cross-section e tempo). O efeito fixo de cross-section é utilizado para capturar as características não observáveis invariantes no tempo, enquanto que o efeito fixo de tempo é utilizado para capturar elementos comuns a todas as empresas que são invariantes ao longo tempo.

A Tabela 3 apresenta os principais resultados. Os modelos revelam um alto ajuste (R-quadrado) para as variáveis dependentes LAJIR e valor de mercado, porém para com o Market to book teve um baixo coeficiente de determinação.

Tabela 3

Regressão com Dados em Painel e Duplo Efeito Fixo Ajustada pela Ferramenta Robust

\begin{tabular}{lcccccc} 
& \multicolumn{2}{c}{ LAJIR } & \multicolumn{2}{c}{ Valor de Mercado } & \multicolumn{2}{c}{ Market to Book } \\
\hline \multirow{2}{*}{ Bse } & $-0,087$ & Significância & Beta & Significância & Beta & Significância \\
crise & 0,520 & 0,098 & 0,279 & 0,103 & 0,155 \\
isexcrise & 0,007 & 0,902 & $-0,450$ & 0,000 & $-0,403$ & 0,000 \\
lnwativo & $-0,210$ & 0,150 & 0,004 & 0,963 & 0,089 & 0,229 \\
lnwliquidez & 0,338 & 0,008 & 0,385 & 0,000 & $-0,154$ & 0,094 \\
lnwdiv_at & 0,032 & 0,023 & 0,053 & 0,000 & 0,017 & 0,226 \\
& $-0,044$ & 0,281 & $-0,113$ & 0,001 & 0,001 & 0,973
\end{tabular}


Tabela 3 (continuação)

\begin{tabular}{lcccccc} 
& \multicolumn{2}{c}{ LAJIR } & \multicolumn{2}{c}{ Valor de Mercado } & \multicolumn{2}{c}{ Market to Book } \\
\cline { 2 - 7 } Inwreceita & 0,254 & 0,002 & 0,080 & 0,046 & 0,160 & 0,053 \\
& $\mathrm{R}^{2}=$ & 0,712 & $\mathrm{R}^{2}=$ & 0,817 & $\mathrm{R}^{2}=$ & 0,151 \\
& $\mathrm{~F}(12,294)=$ & 13,200 & $\mathrm{~F}(12,293)=$ & 60,960 & $\mathrm{~F}(12,291)=$ & 37,980 \\
& Prob $>\mathrm{F}=$ & 0,000 & Prob $>\mathrm{F}=$ & 0,000 & Prob $>\mathrm{F}=$ & 0,000 \\
Efeito Fixo & Cross -Section & $\mathrm{Sim}$ & & $\mathrm{Sim}$ & & $\mathrm{Sim}$ \\
Efeito Fixo & Tempo & $\mathrm{Sim}$ & & $\mathrm{Sim}$ & & $\mathrm{Sim}$ \\
& obs. & 5113 & obs. & 4978 & obs. & 4899 \\
\hline
\end{tabular}

Nota. Fonte: Elaborada pelos autores.

A variável principal a ser focalizada é a de interação entre as variáveis ise e crise. O coeficiente beta desta variável indica a diferença de performance entre as empresas que estão no ise, grupo de tratamento, comparadas com as que não fazem parte do ise, grupo de controle, para o período de crise. Como se pode observar, o coeficiente desta variável não é estatisticamente significante em nenhum dos três modelos. Esse resultado indica que estar listado na carteira ISE-BOVESPA não fez nenhuma diferença em períodos de crise, mesmo que tenham investido recursos na adequação de seus processos e relatórios aos critérios de inclusão da carteira.

Assim, como a participação da empresa no ISE-BOVESPA é a proxy de prática de Responsabilidade Social Corporativa nesta análise, infere-se que não há evidências sobre seu benefício de curto prazo que se está focalizando, isto é, que a prática de RSC traz efeitos positivos na performance financeira da empresa em períodos de crise.

Tais resultados estão alinhados à teoria de Friedman (1970), pois, ao direcionar recursos para adequação aos critérios de inclusão na seleta carteira que assinala comportamentos de Responsabilidade Social Corporativa, as empresas não obtiveram melhores ganhos ou menores perdas para seus acionistas em período de crise econômica. Os resultados também são evidências contra Orlitzky et al. (2003), que argumentam que investimentos em RSC são capazes de fortalecer as firmas para enfrentarem períodos de crise, pelo menos no que diz respeito às condições financeiras.

Para dar credibilidade ao resultado principal, podemos observar que a relação das demais variáveis está totalmente alinhada com a teoria. A variável indicativa do período da crise apresentou significância estatística, impactando negativamente a performance das ações com um decréscimo de 0,4\%, em média, no market to book (lnwmvbv). Tal resultado indica que o delineamento do período de dois trimestres está coerente com os resultados da regressão.

Com relação ao tamanho da empresa, para um aumento de $1 \%$ em seu ativo (lnwativo), o lucro antes dos juros e imposto de renda (lnwlajir) aumenta, em média, 0,34\%. Sendo, portanto, o lucro positivamente sensível ao tamanho da empresa. A liquidez de mercado (lnwliquidez) impacta positivamente e acarreta um aumento no lucro de $0,03 \%$ em média. A variável receita líquida (lnwreceita), quando aumenta em 1\%, acarreta no LAJIR um acréscimo, em média, de 0,25\%.

A dívida bruta sobre o ativo (lnwdiv_at) afetou negativamente o valor de mercado (lnwvm), reduzindo em $0,11 \%$. A variável receita líquida (lnwreceita), quando aumenta em $1 \%$, também, impacta positivamente e causa um acréscimo, em média, de $0,08 \%$. O período assinalado para a crise, apesar de relativamente curto (dois trimestres), foi negativamente impactante no valor de mercado (lnwvm) das empresas, desse modo, representando um decréscimo de $0,45 \%$ em média.

Por fim, a variável ISE, que indica o grupo de empresas que está no Índice de Sustentabilidade ISE-BOVESPA, não apresenta efeito significativo em relação aos três modelos. Isso pode indicar duas 
coisas: a primeira é que de fato RSC não tem efeito sobre o desempenho da empresa; ou que o indicador ISE-BOVESPA não é uma boa proxy para a prática de RSC.

\section{Conclusão}

O objetivo principal deste estudo foi verificar se existe correlação entre Desempenho Social Corporativo e Desempenho Financeiro Corporativo. Para isso, levantaram-se os dados das empresas ativas na Bovespa e fez-se a segregação dos dados das empresas que pertencem ao ISE-BOVESPA daquelas que não pertencem. A partir daí realizou-se a comparação dos dados entre os dois grupos de empresas. Os testes empíricos demonstraram que, em cenários de crise econômica, estar listado no ISEBOVESPA não traz nenhuma alteração no desempenho financeiro das empresas, pois os resultados foram estatisticamente insignificantes para este fator.

Se para Orlitzky et al. (2003), investimentos em RSC são recursos idiossincráticos capazes de fortalecer as firmas para enfrentarem períodos de crise, o presente estudo revela resultados contrários a tal assertiva. Lang e Maffett (2011) encontraram correlação positiva entre a liquidez e valor da empresa e a prática transparência na gestão da empresa. Os resultados foram significativos principalmente no período de crise, quando tais ações tiveram muito melhor desempenho que as demais. Um dos critérios de inclusão no ISE-BOVESPA é a transparência nos relatórios, por isso, esperava-se que por conta desta característica essas ações também obtivessem melhores resultados, porém, com base na amostra deste estudo, tal expectativa não se confirmou.

Neste estudo, foram observadas todas as empresas ativas da Bovespa, totalizando 378 empresas, o que faz o grupo de tratamento (as empresas listadas no ISE) representar um baixo percentual do total da amostra - cerca de $8 \%$ no período. Sugere-se para estudos futuros que seja coletada uma amostra balanceada com números iguais de observações nos grupos de tratamento e de controle, assim como uma coleta discriminatória por setor de operação da empresa.

Entre as limitações deste estudo está o fato de se restringir ao mercado brasileiro de empresas de capital aberto presentes na Bovespa, bem como a apenas uma crise. Como a crise é um fenômeno cíclico e de impacto em diferentes países, sugere-se também a elaboração de estudos que analisem o desempenho de outras carteiras sinalizadoras de RSC presentes nas Bolsas de Valores de outros países e outras crises observadas ao longo das últimas décadas. Tal fato possibilitaria amostra de um maior período e mais empresas, contemplando, assim, entre outros fatores, características institucionais locais.

\section{Agradecimentos}

Os autores agradecem à FAPES - Fundação de Amparo à Pesquisa e Inovação do Espírito Santo pelo apoio proporcionado ao projeto de pesquisa que originou este artigo.

\section{Nota}

\footnotetext{
${ }^{1} \mathrm{O}$ procedimento winsor é uma transformação estatística que limita os valores extremos a um determinado nível de forma a reduzir o efeito espúrio de outliers. Aqui, limitaram-se os valores $5 \%$ inferiores e superiores a um valor igual ao percentil 2,5 e 97,5 .
} 


\section{Referências}

Banerjee, A. V., Gertler, P. J., \& Ghatak, M. (2002). Empowerment and efficiency: tenancy reform in West Bengal. Journal of Political Economy, 110(2), 239-280. doi: 10.1086/338744

BM\&FBOVESPA. (n.d.). Índice de Sustentabilidade Empresarial - ISE. Recuperado de http://www.bmfbovespa.com.br/indices/ResumoIndice.. spx $?$ Indice $=I S E \& O p c a 0=0 \&$ idioma $=p t$ -br

Byus, K., Deis, D. R., \& Ouyang, B. (2010). Doing well by doing good: corporate social responsibility and profitability. SAM Advanced Management Journal, 75(1), 44-55.

Carroll, A. B. (1979). A three-dimensional conceptual model of corporate performance. Academy of Management Review, 4(4), 497-505. doi: 10.5465/AMR.1979.4498296

Cochran, P. L., \& Wood, R. (1984). Corporate social responsibility and financial performance. Academy of Management Journal, 27(1), 42-56. doi: 10.2307/255956

Coelho, C. A., De Mello, J. M. P., \& Funchal, B. (2012). The Brazilian payroll lending experiment. The Review of Economics and Statistics, 94(4), 925-934. doi: 10.1162/REST_a_00228

Cornell, B., \& Shapiro, A. C. (1987). Corporate stakeholders and corporate finance. Financial Management, 16(1), 5-14. doi: 10.2307/3665543

Coulibaly, B., Sapriza, H., \& Zlate, A. (2013). Financial frictions, trade credit, and the 2008-09 global financial crisis. International Review of Economics \& Finance, 26, 25-38. doi: 10.1016/j.iref.2012.08.006.

Donaldson, T., \& Preston, L. E. (1995). The stakeholder theory of the corporation: concepts, evidence and implications. Academy of Management Review, 20(1), 65-91. doi: 10.5465/AMR.1995.9503271992

Du, S., Bhattacharya, C. B., \& Sen, S. (2010). Maximizing business returns to corporate social responsibility (CSR): the role of CSR communication. International Journal of Management Review, 12(1), 8-19. doi: 10.1111/j.1468-2370.2009.00276.x

Fama, E. F. (1970). Efficient capital markets: a review of theory and empirical work. The Journal of Finance, 25(2), 383-417. doi: 10.1111/j.1540-6261.1970.tb00518.x

Freeman, R. E. (2010). Strategic management: a stakeholder approach. Cambridge, UK: Cambridge University Press.

Friedman, M. (1970, September 13). The social responsibility of business is to increase its profits. New York Times Magazine, 13, 122-126.

Galiani, S., Gertler, P., \& Schargrodsky, E. (2005). Water for life: the impact of the privatization of water services on child mortality. Journal of Political Economy, 113(1), 83-120. doi: $10.1086 / 426041$

Gomes, S. F., Jr., \& Gomes, A. R. (2010). As vantagens da sustentabilidade empresarial. Inovação, Gestão e Produção, 2(8), 62-71.

Gowan, P. (2009). Crisis in the heartland consequences of the new Wall Street system. Estudos Avançados, 23(65), 49-72. doi: 10.1590/S0103-40142009000100004

Haggard, S., \& Williamson, J. (1994). The political conditions for economic reform, the political economy of policy reform. Washington DC: Institute for International Economics. 
Ivashina, V., \& Scharfstein, V. (2010). Bank lending during the financial crisis of 2008. Journal of Financial Economics, 97(3), 319-338. doi: 10.1016/j.jfineco.2009.12.001

Lal, R., Miller, S., Lieuw-Kie-Song, M., \& Kostzer, D. (2010). Public works and employment programmes: towards a long-term development approach [Working Paper $\mathrm{n}^{\circ}$ 66]. International Policy Centre for Inclusive Growth, Brasília, DF, Brasil. Recuperado de http://www.ipcundp.org/pub/IPCWorkingPaper66.pdf

Lang, M., \& Maffett, M. (2011). Transparency and liquidity uncertainty in crisis periods. Journal of Accouting and Economics, 52(2/3), 101-125. doi: 10.1016/j.jacceco.2011.07.001

Lee, D. D., \& Faff, R. W. (2009). Corporate sustainability performance and idiosyncratic risk: a global perspective. The Financial Review, 44(2), 213-237. doi: 10.1111/j.1540-6288.2009.00216.x

López, M. V., Garcia, A., \& Rodriguez, L. (2007). Sustainable development and corporate performance: a study based on the Dow Jones sustainability index. Journal of Business Ethics, 75(3), 285-300. doi: 10.1007/s10551-006-9253-8

Machado, M. R., Machado, M. A. V., \& Corrar, L. J. (2009). Desempenho do Índice de Sustentabilidade Empresarial (ISE) da Bolsa de Valores de São Paulo. Revista Universo Contábil, 5(2), 24-38. doi: 10.4270/ruc.2009211

Mackey, A., Mackey, T. B., \& Barney, J. B. (2007). Corporate social responsibility and firm performance: investor relations and corporate strategies. Academy of Management Review, 32(3), 817-835. doi: 10.5465/AMR.2007.25275676

Mallin, C., Faraga, H., \& Ow-Yong, K. (2014). Corporate social responsibility and financial performance in Islamic banks. Journal of Economic Behavior \& Organization, 103(Suppl.), S21S38. doi: 10.1016/j.jebo.2014.03.001

Marcondes, A. W., \& Bacarji, C. D. (2010). ISE - Sustentabilidade no mercado de capitais. São Paulo: Report.

McWilliams, A., \& Siegel, D. (2001). Corporate social responsibility: a theory of the firm perspective. The Academy of Management Review, 26(1), 117-127. doi: 10.5465/AMR.2001.4011987

Meyer, B. D. (1995). Natural and quasi-experiments in economics. Journal of Business \& Economic Statistics, 13(2), 151-161. doi: 10.2307/1392369

Minsky, H. P. (1986). Stabilizing an unstable economy. New York: McGraw Hill Professional. Retrieved from http://digamo.free.fr/minsky86.pdf

Mizen, P. (2008). The credit crunch of 2007-2008: a discussion of the background, market reaction, and policy responses. Federal Reserve bank of St Louis Review, 90(5), 531-567.

Moskowitz, M. (1972). Choosing socially responsible stocks. Business and Society Review, 1, 71-75.

Orlitzky, M., Schmidt, F. L., \& Rynes, S. L. (2003). Corporate social and financial performance: a metaanalysis. Organizational Studies, 24(3), 403-441. doi: 10.1177/0170840603024003910

Pavie, J. J. E. (2008). Responsabilidade social corporativa e desempenho financeiro: uma meta-análise (Dissertação de mestrado). Programa de Pós-Graduação em Administração, Faculdades Ibmec, Rio de Janeiro, RJ, Brasil.

Poddi, L., \& Vergalli, S. (2009). Does corporate social responsibility affect the performance of firms? [Working Paper n ${ }^{\circ}$ 313]. Fondazione Eni Enrico Mattei Working Papers. Retrieved from http://www.bepress.com/feem/paper313 
Porter, M. E., \& Linder, C. V. (1995). Green and competitive: ending the stalemate. Harvard Business Review, 73(5), 120-134.

Rose, A. K., \& Spiegel, M. M. (2012). Cross-country causes and consequences of the 2008 crisis: early warning. Japan and the World Economy, 24(1), 1-16. doi:10.1016/j.japwor.2011.11.001

Servaes, H., \& Temayo, A. (2013). The impact of corporate social responsibility on firm value: the role of customer awareness. Management Science, 59(5), 1045-1061. doi: 10.1287/mnsc.1120.1630

Spence, M. (1973). Job market signaling. The Quarterly Journal of Economics, 87(3), 355-374. doi: $10.2307 / 1882010$

Tang, Z., Hull, C., \& Rothenberg, S. (2012). How corporate social responsibility engagement strategy moderates the CSR-Financial Performance Relationship. Journal of Management Studies, 49(7), 1274-1303. doi: 10.1111/j.1467-6486.2012.01068.x

Teixeira, E. A., Nossa, V., \& Funchal, B. (2011). O índice de sustentabilidade empresarial (ISE) e os impactos no endividamento e na percepção de risco. Revista Contabilidade Financeira, 22(55), 29-44. Recuperado de http://www.scielo.br/pdf/rcf/v22n55/a03v22n55.pdf. doi: 10.1590/S151970772011000100003

Vance, S. C. (1975). Are socially responsible corporations good investment risks. Management Review, 64(8), 18-24.

Waddock, S. A., \& Graves, S. B. (1997). The corporate social performance-financial performance link. Strategic Management Journal, 18(4), 303-319. doi: 10.1002/(SICI)10970266(199704)18:4<303::AID-SMJ869>3.0.CO;2-G

Wagner, T., Lutz, R. J., \& Weitz, B. A. (2009). Corporate hypocrisy: overcoming the threat of inconsistent corporate social responsibility perceptions. Journal of Marketing, 73(6), 77-91. doi: 10.1509/jmkg.73.6.77

Waller, D. S., \& Lanis, R. (2009). Corporate social responsibility (CSR) disclosure of advertising agencies: an exploratory analysis of six holding companies annual reports. Journal of Advertising, 38(1), 109-121. doi: 10.2753/JOA0091-3367380107

Wang, T., \& Bansal, P. (2012). Social responsibility in new ventures: profiting from a long-term orientation. Strategic Management Journal, 33(10), 1135-1153. doi: 10.1002/smj.1962

Wang, W. K., Lu, W. M., Kweh, Q. L., \& Lai, H. W. (2014). Does corporate social responsibility influence the corporate performance of the U.S. telecommunications industry? Telecommunications Policy, 38(7), 580-591. doi: 10.1016/j.telpol.2014.01.004

\section{Dados dos Autores}

\section{Lilian Marques Freguete}

Av. Fernando Ferrari, 1358, 29075-505, Boa Vista, Vitória, ES, Brasil. E-mail: lilian.freguete@ hotmail.com

Valcemiro Nossa

Av. Fernando Ferrari, 1358, 29075-505, Boa Vista, Vitória, ES, Brasil. E-mail: valcemiro@ fucape.br

Bruno Funchal

Av. Fernando Ferrari, 1358, 29075-505, Boa Vista, Vitória, ES, Brasil. E-mail: bfunchal@ fucape.br 